\title{
Mechanical Characteristics of Rat Vibrissae: Resonant Frequencies and Damping in Isolated Whiskers and in the Awake Behaving Animal
}

\author{
Mitra J. Hartmann, Nicholas J. Johnson, R. Blythe Towal, and Christopher Assad \\ Jet Propulsion Laboratory, California Institute of Technology, Pasadena, California 91109
}

\begin{abstract}
We investigated the natural resonance properties and damping characteristics of rat macrovibrissae (whiskers). Isolated whiskers rigidly fixed at the base showed first-mode resonance peaks between 27 and $260 \mathrm{~Hz}$, principally depending on whisker length. These experimentally measured resonant frequencies were matched using a theoretical model of the whisker as a conical cantilever beam, with Young's modulus as the only free parameter. The best estimate for Young's modulus was $\sim 3-4 \mathrm{GPa}$. Results of both vibration and impulse experiments showed that the whiskers are strongly damped, with damping ratios between 0.11 and 0.17 . In the behaving animal, whiskers that deflected past an object were observed to resonate but were damped significantly more than isolated whiskers. The time course of damping varied depending on the individual whisker and the phase of the whisking cycle, which suggests that the rat may modulate biomechanical parameters that affect damping. No resonances were observed for whiskers that did not contact the object or during free whisking in air. Finally, whiskers on the same side of the face were sometimes observed to move in opposite directions over the full duration of a whisk. We discuss the potential roles of resonance during natural exploratory behavior and specifically suggest that resonant oscillations may be important in the rat's tactile detection of object boundaries.
\end{abstract}

Key words: vibrissae; whiskers; rat; tactile; tactual; resonance; vibration; exploratory behavior; exploration; whisking; edge detection

\section{Introduction}

Rats use active movements of their vibrissae (whisking) to extract information about the spatial properties of objects, including size, shape, and texture (Vincent, 1913; Welker, 1964; Carvell and Simons, 1990, 1995). Because whisking typically occurs at frequencies between 5 and $15 \mathrm{~Hz}$ (Welker, 1964; Berg and Kleinfeld, 2003), many studies have characterized neuronal responses to whisker stimulation or active movement in this frequency range (Zucker and Welker, 1969; Shipley, 1974; Simons, 1985; Hartings and Simons, 1998; Hartmann and Bower, 1998; Ahissar et al., 2000; Sosnik et al., 2001; Castro-Alamancos, 2002; Kleinfeld et al., 2002; O’Connor et al., 2002).

Several studies have additionally suggested that neurons in the trigeminal pathway may respond to whisker deflections at frequencies much higher than $15 \mathrm{~Hz}$. Specifically, mechanoreceptors in the sinus hair follicle respond at frequencies up to $1500 \mathrm{~Hz}$ (Gottschaldt and Vahle-Hinz, 1981), and cells in the trigeminal ganglion respond to high frequency vibration up to $1000 \mathrm{~Hz}$ (Gibson and Welker, 1983a,b). More central levels of the trigeminal system show robust

\footnotetext{
Received March 4, 2003; revised May 29, 2003; accepted May 30, 2003.

This work was supported by NASA's Information Technology Strategic Research Program. We are grateful to Terry Scharton, whose expertise on mechanical vibrations helped guide this work. We also thank Steve Lewis in the Jet Propulsion Laboratory standards laboratory for training and assistance on the use of the vibration table and Maria Neimark for useful discussions.

Correspondence should be addressed to Dr. Mitra J. Hartmann, Jet Propulsion Laboratory, Mail Stop 303-300, California Institute of Technology, 4800 0ak Grove Drive, Pasadena, CA 91109. E-mail: mitra.hartmann@jpl.nasa.gov.

Copyright $\odot 2003$ Society for Neuroscience $\quad 0270-6474 / 03 / 236510-10 \$ 15.00 / 0$
}

responses at least to $40 \mathrm{~Hz}$ (trigeminal nuclei) (Shipley, 1974) (thalamus) (Castro-Alamancos, 2002) (somatosensory cortex) (Simons, 1978; Kleinfeld et al., 2002), but generally have not been tested at higher frequencies. Thus, there are hints that cells at many levels of the trigeminal system may be responsive to frequencies far above typical whisking ranges.

One problem in identifying the frequency ranges of relevance to the vibrissal system is that the mechanics of whisker transduction have not yet been characterized; we do not have a quantitative description of how mechanical stimuli are generated during natural whisking behaviors, transmitted to sensory cells in the whisker follicle, and coupled to the rat's nervous system. Recently, Neimark proposed that high-frequency whisker resonances may aid in texture discrimination (Neimark, 2001; Neimark et al., 2003). In the current study, we performed vibration and impulse experiments on isolated whiskers and compared the results with whisker oscillations observed during exploratory behaviors in the awake animal. The whiskers exhibited natural resonance frequencies and damping that were well matched using a model of the whisker as a conical cantilever beam. Our results suggest that natural resonance properties of the whiskers contribute to vibrational stimuli during the initial transient responses that occur immediately after a whisker deflects past an object, which may be particularly important for the detection of object boundaries. Increases in damping seen under particular behavioral conditions also suggest that the rat may be able to actively modulate the resonant frequencies. This work represents our first step toward modeling whisker movements and predicting the 


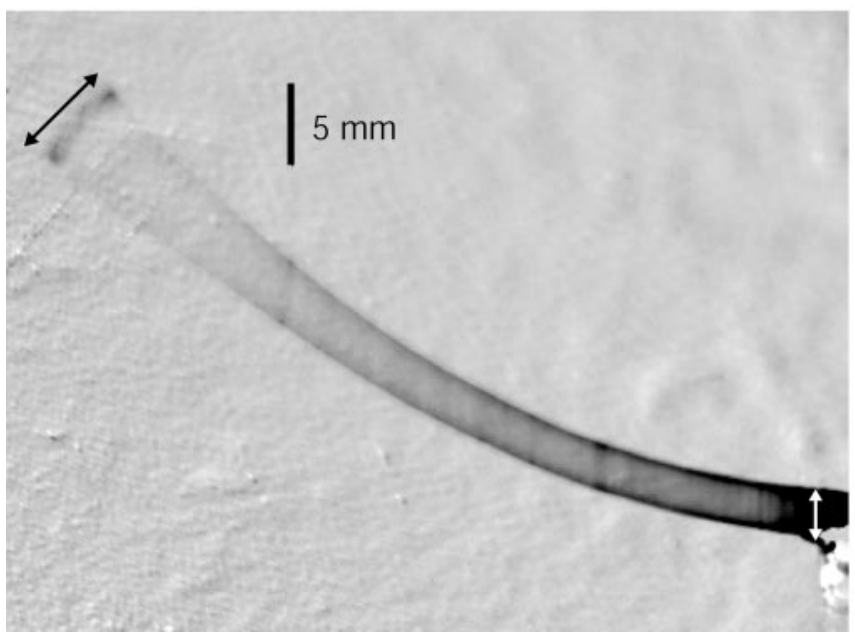

Figure 1. Measurement of tip displacement in a vibrating whisker. The displacement of the tip is indicated by the black arrow, and the displacement of the base by the white arrow. The picture is black-and-white reversed. The tip is illuminated by a laser line, and the amplitude of tip motion is measured between the two dark extrema.

pattern of peripheral input generated during the rat's active sensing behaviors.

\section{Materials and Methods}

Whisker measurements. Whiskers were obtained from an adult, female Sprague Dawley rat that had been killed in an unrelated experiment. We examined a total of 24 whiskers: $\alpha, \beta, \gamma$, and $\delta$, and the first four whiskers of each of rows A through E. Each whisker was grasped firmly at its base with fine forceps and plucked out of its follicle.

The mass of each whisker was measured on a Cahn-35 microbalance (Orion Research, Beverly, MA). The length of each whisker was measured with a ruler under a standard fluorescent magnifying lamp. The base and tip radii of each whisker were measured under a Leitz (Wetzlar, Germany) microscope. These measurements allowed us to calculate the whisker volume, assuming a truncated cone, and the density for each whisker was then determined by dividing its mass by its volume. This method of calculating whisker density is very sensitive to small errors in measurement of the radius of the base. We therefore used the average density over all of the whiskers $\left(1.14 \mathrm{mg} / \mathrm{mm}^{3}\right)$ to predict the theoretical resonance frequencies (see Results). All of the procedures were approved in advance by the Animal Care and Use Committee of the California Institute of Technology.

Resonance measurements. A small drop of superglue was used to fix each whisker, in turn, to a computer-controlled vibration table in the standards laboratory at the Jet Propulsion Laboratory. Because the whiskers had an inherent curvature, each whisker was always mounted concave up (Fig. 1). Whiskers were illuminated with ambient light, and a linear laser beam was oriented along the direction of whisker motion at its tip. We videotaped the movement of each whisker on the vibration table using two high-speed digital video cameras (Photron Fastcams; Photron, San Diego, CA). One camera was mounted directly above the whisker (obtaining a bird's-eye view), while the second camera was mounted to view the vertical motion of the length of the whisker (longitudinal view). The vibration table moved only along the vertical axis, and, therefore, the longitudinal camera captured the whisker motion in the vertical plane.

Because we first wanted to evaluate the motion blur of the whisker (indicating the fullest extent of whisker displacement), the cameras were not used in high-speed mode in initial experiments but rather were set to take data at 30 frames/sec. During initial data analysis, we found that the motion of the whisker in the overhead camera view was negligible in comparison with its motion in the longitudinal camera view, and we therefore considered only the motion in the vertical plane.

A resonance curve for each whisker was obtained by driving the whis- ker at a wide range of different frequencies $(f)$ using constant acceleration. Constant acceleration is standard engineering practice and means that the amplitude of vibration at the base is inversely proportional to $(2 \pi f)^{2}$. In practice, constant acceleration $( \pm 0.5 \%)$ proved feasible down to $25 \mathrm{~Hz}$.

All of the whiskers studied had first-mode resonant frequencies of $\geq 27$ Hz. For particularly long whiskers, we constructed resonance curves that included frequencies as low as $10 \mathrm{~Hz}$ (see Fig. 4). At frequencies of $<25$ $\mathrm{Hz}$, we measured the acceleration of the table, and then scaled the data appropriately (using Eq. 1) to match the constant acceleration obtainable at frequencies of $\geq 25 \mathrm{~Hz}$.

The peak acceleration of the table $(\alpha)$ was measured in units of $g$, the acceleration of gravity $\left(9.8 \mathrm{~m} / \mathrm{sec}^{2}\right)$. At each frequency $(f)$, the amplitude of the base displacement was thus calculated as follows:

$$
A_{\text {base }}=(\alpha)(9.8) /(2 \pi f)^{2}
$$

By using a wide range of frequencies, we were prepared to observe multiple resonance modes of the whisker. We generally began by increasing frequency of the vibration table in $10-20 \mathrm{~Hz}$ intervals and then narrowed in to $2 \mathrm{~Hz}$ intervals once the approximate resonances had been found. Between 100 and 500 frames of video data were recorded at each frequency, and each frame was saved as a TIFF file $(640 \times 480$ pixels $)$. At a frame rate of 30 frames/sec (shutter open), the whisker appears as a blur, and the image indicates the full range of motion. Extreme positions of whisker motion appear darker, because the whisker spends a longer time in these positions (the whisker velocity is zero). At the end of each run (covering the full frequency range), we calibrated the image by taking videos while a ruler was held parallel to and then perpendicular to the whisker. The whisker was then removed from the vibration table, and 500 frames of video data were taken of the background.

The frames of video data taken at each frequency were next imported into Matlab (The MathWorks, Nattick, MA) and averaged. The frames of the background image were also imported into Matlab and averaged. We then subtracted the average background image from the average image of the (blurred) whisker motion at each frequency. For each frequency, we then directly measured the tip displacement, as shown in Figure 1.

Dividing the tip displacement by the base displacement at each measured frequency yields a magnification ratio curve, which is useful in characterizing resonances. In principle, the base displacement could have been directly measured from an image such as Figure 1. However, it is difficult to obtain an accurate measure of base amplitude by this technique at higher frequencies (see Fig. 3, middle row). Therefore, base peak-to-peak displacement $\left(2^{*} A_{\text {base }}\right)$ was directly calculated from Equation 1.

Damping measurements. To confirm our values for the first-mode resonance frequencies and also to investigate the damping characteristics of the whiskers, we performed impulse experiments on the four whiskers of row D. For these experiments, the whisker remained superglued to the vibration table, but the acceleration was turned off. The vibration table was isolated from the external vibrations in the environment.

We used a pair of fine forceps held by hand to deflect the whisker near its base. The forceps were initially placed above the whisker and then rapidly moved in a straight downward direction past the whisker to achieve an initial displacement with minimum initial velocity (plucking). Care was taken to deflect the whisker in only one direction and to ensure that the forceps were not in contact with the whisker after the initial displacement.

We again used the Photron Fastcams to monitor the displacement of the whisker in two dimensions. This time, however, video was taken at 1000 frames/sec with a shutter speed of $200 \mu \mathrm{sec}$. Again, motion of the whisker in the overhead camera view was found to be negligible. The video images were imported into Matlab, and the position of the whisker was manually tracked. For each frame, we determined the angle that the whisker tip made with respect to the horizontal axis as the whisker oscillated (in its first-mode resonance) back to its static position. These angle measurements were low-pass filtered at more than two times the fundamental resonance frequency for smoothness, and the peaks and troughs of the oscillations were then located. 
Table $1 . I_{\mathrm{n}}$ values used in the theoretical model to predict resonant frequencies for the fixed-free conical beam

\begin{tabular}{llll}
\hline$\left(A_{t} / A_{b}\right)^{1 / 2}$ & Mode 1 & Mode 2 & Mode 3 \\
\hline 1.00 & 3.516 & 22.034 & 61.701 \\
0.500 & 4.625 & 19.55 & 48.50 \\
0.333 & 5.289 & 18.76 & 43.78 \\
0.250 & 5.850 & 18.51 & 41.34 \\
0.100 & 7.201 & 18.71 & 37.10 \\
0 & 8.718 & 21.146 & 38.45 \\
\hline
\end{tabular}

Values are from Table 1 by Georgian (1965). Our values were interpolated from this table to adjust for measured tip and base diameter. $A_{t}$ is the area of the tip of the conical cantilever beam, and $A_{b}$ is the area of the base.

To find the damping ratio, we plotted the log of the amplitudes of the peaks and troughs of the oscillation versus the number of oscillation cycles. The slope of this line is the logarithmic decrement $\Delta$. The logarithmic decrement is then related to the damping ratio $\zeta$ as follows (Tongue, 2002):

$$
\Delta=2 \pi \zeta /\left(1-\zeta^{2}\right)^{1 / 2}
$$

so that in the underdamped case,

$$
\zeta=\Delta /\left(\Delta^{2}+4 \pi^{2}\right)^{1 / 2}
$$

A second value for $\zeta$ was calculated by dividing the bandwidth of the resonance curve at the half-power $(-3 \mathrm{~dB})$ point by twice the resonance frequency (Tongue, 2002). These resonance curves were those obtained in the previous experiment and provide an independent measurement of $\zeta$. Finally, the quality factor $(Q)$ is defined as $1 / 2 \zeta$ (Tongue, 2002).

Theoretical prediction of natural frequencies. Neimark first proposed using a cylindrical cantilever beam to model the vibrations of the whiskers (Neimark, 2001). Here, we extend the model to a truncated conical cantilever beam. According to theory (Conway et al., 1964), a tapered conical beam fixed at one end and free to vibrate at the other end will have resonant frequencies $f_{n}$ equal to

$$
f_{n}=\left(\lambda_{n} / L^{2}\right)\left(E I / \rho A_{b}\right)^{1 / 2}
$$

where $\lambda_{n}$ are tabulated constants determined by boundary conditions, $L$ is the length of the cone, $E$ is Young's modulus, $A_{b}$ is the cross-sectional area at the base of the cone, $I$ is the cross-section moment of inertia at the base of the cone $\left(A_{b}^{2} / 4 \pi\right)$, and $\rho$ is the density of the material. The constants $\lambda_{n}$ are determined by fixed-free boundary conditions and by the amount of tapering from base to tip. The numerical model of Conway et al. (1964) was verified experimentally by Georgian (1965), who tabulates $\lambda_{n}$ in terms of $\left(A_{t} / A_{b}\right)^{1 / 2}$, where $A_{t}$ is the area at the tip of the cone. We used these values for $\lambda_{n}$ in our model to account for tip diameter, as shown in Table 1. As described above, we used an average value of $\rho$ calculated over all of the whiskers in our theoretical prediction of resonant frequencies. We did not measure Young's modulus of the whiskers. Instead, $E$ was left as a free parameter, and its value was estimated on the basis of the best fit between measured and predicted values (see Results).

Video quantification of natural exploratory behaviors. An adult $(\sim 6$ months) female Sprague Dawley rat was placed in an $11 \times 11$ inch square cage with a sliding door that could be manually opened and closed. At the onset of each trial (indicated by door opening), the rat poked its head and upper body out of the cage and searched for a liquid reward in a pipette. The trial was terminated after the rat located and consumed the reward, and retracted its head back into the cage. All of the procedures were approved in advance by the Animal Care and Use Committee of the California Institute of Technology.

A flat fluorescent light source was positioned under the door, and the Photron Fastcams were used to monitor the head and whisker movements of the rat at either 250 or $1000 \mathrm{frames} / \mathrm{sec}$. One camera was placed directly above the door, obtaining a bird's-eye view, silhouetted image of the rat's head and whiskers (Fig. 2). The second camera was positioned to the side of the animal to monitor head tilt. For the results presented in this paper, we analyzed only those trials in which head tilt was determined to be negligible.
On some trials, a metal "C" was placed in the rat's search space. The " $C$ " consisted of a vertical bar $\sim 12$ inches tall and two horizontal bars positioned well above and below the search space of the rat (Fig. $2 A$ ). The "C" thus effectively appeared as a vertical post to the rat. Consistent with exploration strategies described previously (Brecht et al., 1997; Hartmann, 2001), the rat detected the vertical post with its macrovibrissae and then explored it more closely with its snout and microvibrissae. During the initial detection phase, we were able to examine the macrovibrissal deflections that occurred as the rat brushed its whiskers past the post.

Figure 2 shows representative video frames illustrating our tracking methods for determining angular whisker position during natural exploratory behaviors. For each video segment, we first determined which whiskers could be accurately tracked throughout the entire behavioral sequence. In each video frame, we then tracked the base of these whiskers and also a point further out along the whisker. For videos taken of free whisking behavior, we additionally traced out the entire whisker shape, as shown in Figure $2 B$.

Head and whisker positions were tracked semiautomatically using image-processing scripts written in Matlab. Whisker angles were measured relative to the rat's head angle, with $180^{\circ}$ representing rostral and $0^{\circ}$ representing caudal. When fully protracted, whiskers could attain angles close to $180^{\circ}$ (e.g., see the most rostral whiskers of Fig. $2 \mathrm{~B}$ ). When fully retracted, the minimum angle a whisker could attain was the angle it formed when lying flat back against the head, or $\sim 30^{\circ}$.

\section{Results}

\section{Resonance modes}

We first measured the resonance modes of isolated whiskers by applying a sinusoidal displacement at the whisker base over a range of frequencies (see Materials and Methods). The base of the whisker was superglued to the vibration table, so it was displaced directly with the table. The tip of the whisker was then free to move based on whisker dynamics. At some vibration frequencies, the displacement of the whisker tip was disproportionately large relative to the displacement of the whisker base, indicating that the whisker had entered a resonance mode. Figure 3 shows first-, second-, and third-mode vibrations for the $\mathrm{C} 1$ whisker, at frequencies of 40, 94, and $188 \mathrm{~Hz}$, respectively. The $\mathrm{C} 1$ whisker was $\sim 53 \mathrm{~mm}$ long and had a base radius of $105 \mu \mathrm{m}$ and a tip radius of $5.5 \mu \mathrm{m}$. Note that the spatial position of the node of the second mode is not at the midpoint of the whisker, as would be expected for a cylindrical beam. Instead, the node is much further out along the length of the whisker, consistent with the expected effect of the whisker taper.

Figure 4 illustrates the resonance curve for whisker $\mathrm{C} 1$. The top graph shows the displacement of the tip (solid line) and the base (dashed line) as a function of frequency. The magnification ratio was calculated by dividing the tip displacement by the base displacement and is shown in the bottom graph of Figure 4. Two features of the ratio curve are particularly noteworthy. First, all of the modes are strongly damped, as shown by the fact that the peaks are very broad. In fact, the first mode is so highly damped that it appears only as a cusp on the resonance ratio curve.

Second, the amplitude of the resonance peaks increases with mode. This is a surprising result, because the amplitude of the resonance peaks in typical engineering applications decreases with mode number (Gorman, 1975). We suggest that this result is due at least in part to nonlinear effects of viscous and/or hysteretic damping. Viscous damping, arising from the motion of a body through a fluid, varies with velocity; hysteretic damping, resulting from the stresses placed on an imperfectly elastic material, varies with displacement (Tongue, 2002). Standard engineering analyses of beam vibrations, in which the amplitude of the resonance peaks typically decreases with mode number, usu- 


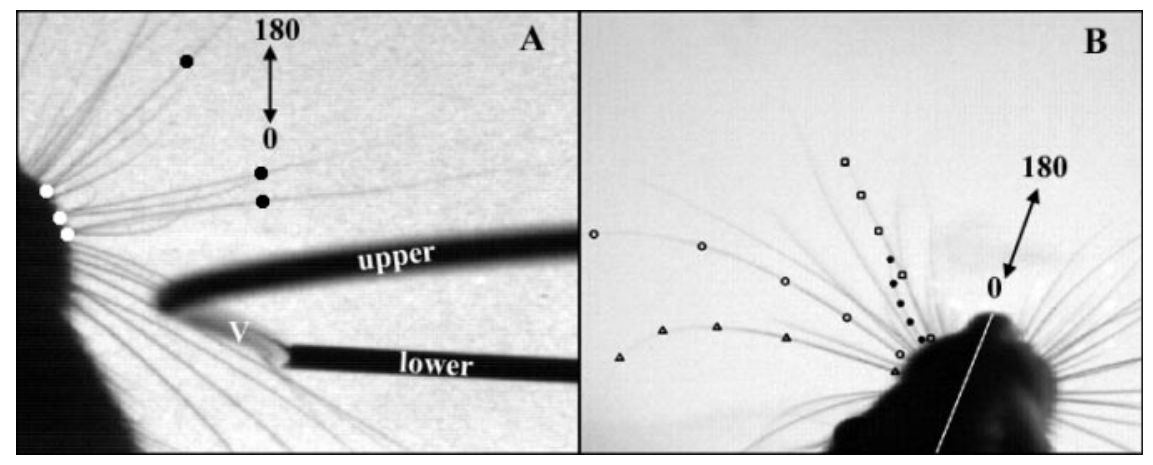

Figure 2. Method for tracking the angular position of whiskers during deflection past an object and during free whisking in air. Whisker angles are measured relative to head angle, with $180^{\circ}$ representing rostral and $0^{\circ}$ caudal. $A$, A single frame from a video of the rat driving its macrovibrissae past a metallic " $C$." The vertical portion of the " $C$ " (labeled " $V$ ") is 12 inches in height and appears foreshortened in the image. The horizontal bar labeled "upper" is well above the rat's head. The horizontal bar labeled "lower" is well below the rat's head. The more caudal whiskers are clearly stuck behind the vertical bar, while the more rostral whiskers are free to move in front of the bar. Whisker angles were defined as the angle formed by the base of the whisker (white circles) and a point some length out along the whisker (black circles), relative to the angle of the head. $B$, The full shape of each whisker was tracked during free whisking behavior. Whisker angles were determined from the angle between the base point and the last point out along the whisker, relative to the head angle. Triangles, filled and open circles, and squares exemplify the tracking of four separate whiskers.
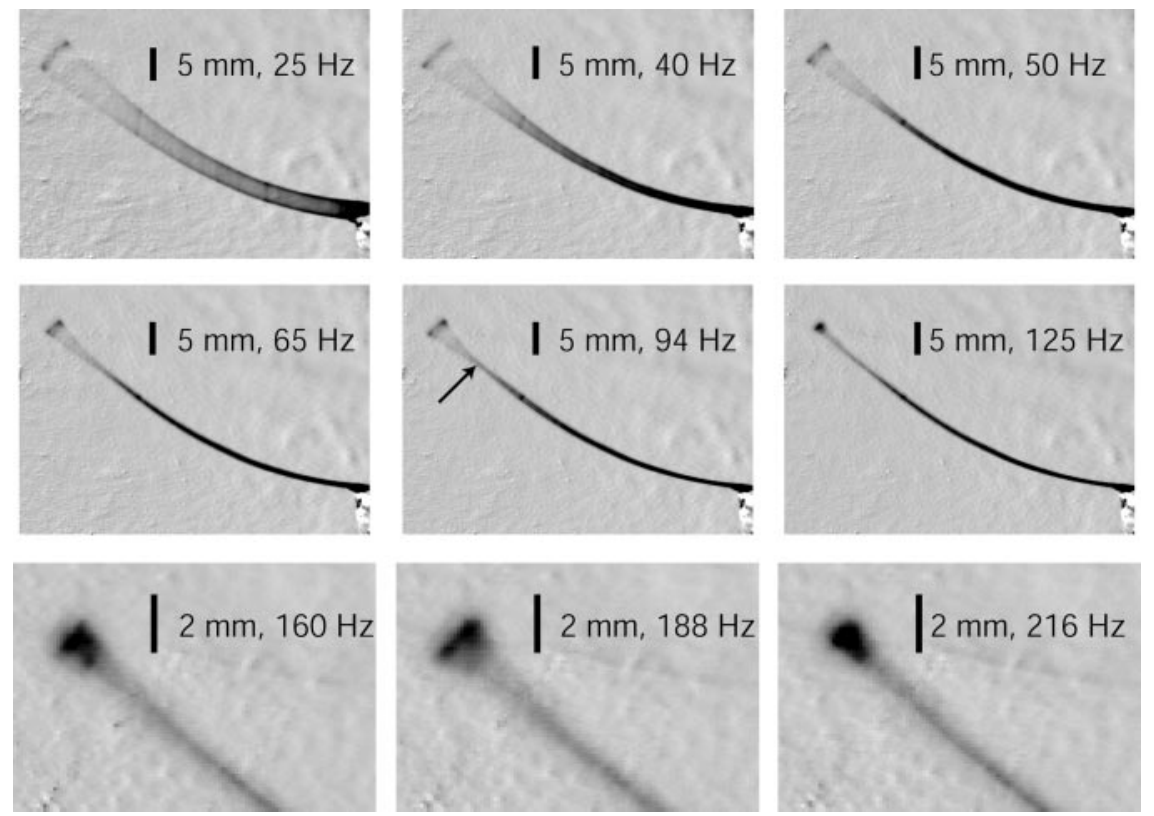

Figure 3. First, second, and third resonant modes for whisker C1. The top row shows the first mode near $40 \mathrm{~Hz}$ and vibration at surrounding frequencies of $25 \mathrm{and} 50 \mathrm{~Hz}$. Note that, although the tip displacement does not appear much larger in the $40 \mathrm{~Hz}$ image than in the $25 \mathrm{~Hz}$ image, the base amplitude is much smaller in the $40 \mathrm{~Hz}$ image. The middle row shows the second mode at $94 \mathrm{~Hz}$ and the vibration at nearby frequencies of 65 and $125 \mathrm{~Hz}$. Note the narrow neck (arrow) at resonance indicating the node of the second mode. The bottom row shows the third mode at $188 \mathrm{~Hz}$ and the vibration at the surrounding values of $160 \mathrm{and} 216 \mathrm{~Hz}$.

ally assume small-amplitude deflections and linear viscous damping. These conditions were unlikely to hold during our experiments. If damping was nonlinear, in either velocity or displacement, then the lower modes would be more strongly damped, because these quantities are greater at lower frequencies for constant acceleration input.

In the behaving animal, the magnification ratio, as shown in Figure 4, is unlikely to be the most relevant parameter for sensory perception. More useful measures may be the ratio of whisker displacement to the force exerted by interactions with external objects, or the fraction of force transmitted to the base from a particular whisker displacement. However, these two measures require determining the forces exerted on a distributed mass, which is difficult even in the isolated whisker preparation and requires a more elaborate experimental apparatus.

\section{Resonance frequencies: experiment versus theory}

Using Equation 4 (Conway et al., 1964; Georgian, 1965), we were able to calculate natural resonances on the basis of measured values of length, density, moment of inertia, and known values of $\lambda_{1}$. The average whisker density over all 24 whiskers was found to be $1.14 \pm 0.27 \mathrm{mg} / \mathrm{mm}^{3}$, and this was therefore the value used to predict the resonant values for the whiskers. Young's modulus was taken to be a free parameter for all 24 whiskers. A comparison between theoretical calculations and measured first resonance frequency is shown in Figure 5. Figure $5 \mathrm{~A}$ illustrates that, over all of the whiskers, the best match between predicted and measured values was found for a value of $E=3.02 \mathrm{GPa}$.

Careful inspection of Figure 5A, however, suggests that a lone outlier at high frequency $(+)$ may have unduly influenced the curve fit between measured and predicted values. This whisker was the $\mathrm{E} 4$ whisker, the shortest one in our analysis. We therefore reanalyzed our data after removing the single highest-frequency value (Fig. 5B). The remaining 23 whiskers gave a much better fit between measured and predicted values, with $E=3.68 \mathrm{GPa}$. This range of values for $E(\sim 3-4 \mathrm{GPa})$ is close to $E$ for keratin material $(2.5 \mathrm{GPa})$ (Bonser and Purslow, 1995) (1.33-1.84 GPa) (Bonser, 2000). In biological materials, Young's modulus is likely to be anisotropic (Bonser, 2000), and thus, the value is at best an approximation. For comparison, $E$ for rubber is $0.0028 \mathrm{GPa}, E$ for nylon is between 2 and $3 \mathrm{GPa}$, and $E$ for stainless steel is between 190 and $200 \mathrm{GPa}$.

Having determined a range of values for $E$, we can now compare the measured values of the resonant frequencies for whisker $\mathrm{C} 1$ above with predicted values. As shown in Figure 4, the measured values for the three first resonant frequencies for the $\mathrm{C} 1$ whisker were 40, 94, and $188 \mathrm{~Hz}$. Assuming $E=3.68 \mathrm{GPa}$, our model of the whisker as a conical beam predicts that the first three resonant modes would occur at 47, 115, and $213 \mathrm{~Hz}$. The measured values for the first three resonant modes of this whisker are thus lower, but within $20 \%$, of the predicted values.

The measured natural frequencies of the whiskers varied inversely with whisker length (Fig. 6A). We next compared these experimental data with the theoretical model relating frequency to length $(L)$. Equation 4 predicts explicitly that the resonant frequencies should decrease inversely as $L^{-2}$. However, a log-log plot of the measured data as a function of length has a slope of 

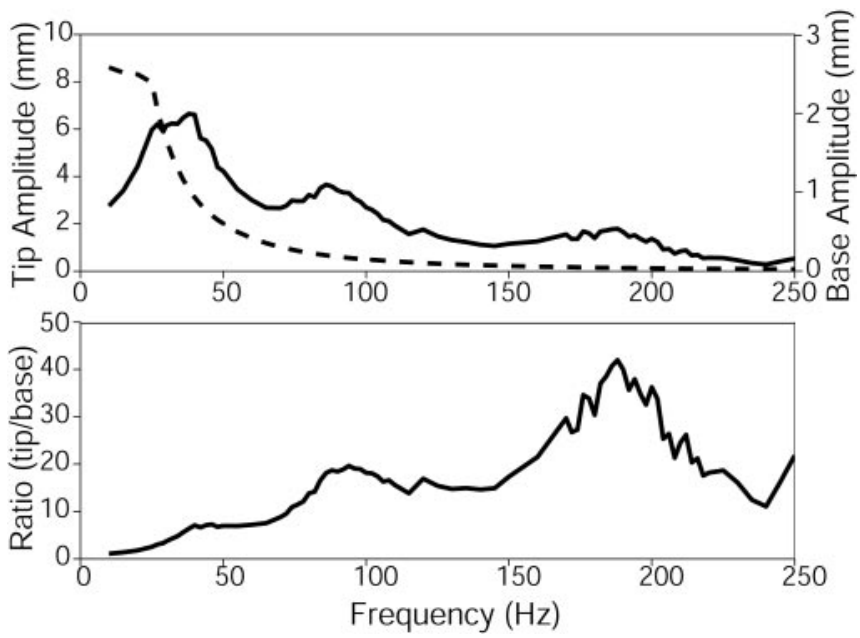

Figure 4. Resonance curve for whisker (1. The top graph shows the absolute displacement of the tip (solid line) and the absolute displacement of the base (dashed line). The vertical scale on the left applies to the tip, whereas the vertical scale on the right applies to the base. The bottom plot shows the magnification ratio curve for the whisker, calculated by dividing the displacement of the tip by the displacement of the base. Resonance peaks are seen near 40,94, and $188 \mathrm{~Hz}$. All of the peaks are broad because of damping.

-1.5 (Fig. $6 B$ ), indicating that the measured frequencies decrease as $L^{-1.5}$, instead of $L^{-2}$. This is because Equation 4 also has an implicit linear dependence on the base radius $\left(R_{b}\right)$ of the whisker. Substituting for $I$ and $A_{b}$, Equation 4 can be restated as:

$$
f_{n}=\left(\lambda_{n} R_{b} / 2 L^{2}\right)(E / \rho)^{1 / 2}
$$

A log-log plot of measured base radii versus whisker length (Fig. $6 C$ ) showed that the radius increases approximately as the square root of the length (the slope of the plot is 0.49). Inserting this relationship into Equation 5 yields a frequency dependence of $L^{0.5} / L^{2}$, which corresponds to the $L^{-1.5}$ observed in Figure $6 B$.

\section{Damping measurements}

We next investigated the damping characteristics of the whiskers by performing impulse experiments on the four whiskers of the D row (see Materials and Methods). This serves as an independent check for the first-mode resonant frequencies and also permits a more detailed analysis of damping coefficients.

In our initial analysis of the impulse experiments, we observed that each whisker oscillated around a position different from its initial static position. In many cases, the whisker did not even return to its original static position after the oscillations ended. We therefore calculated the zero line for the oscillation based on the physical constraint that the amplitude of the oscillation must decrease with each half-cycle (i.e., energy must never be added into the oscillation). In practice, there was only one such line for each impulse trial (to within $0.2^{\circ}$ ), and its value corresponded directly to the midpoint of the last observable cycle in the oscillation.

We analyzed three impulse trials for each of the four whiskers in the $\mathrm{D}$ row. For each whisker, the impulse trials gave first-mode resonance values that agreed to within $5 \mathrm{~Hz}$ of each other, and also within $5 \mathrm{~Hz}$ of the first-mode resonance value as measured in the vibration table experiment. Figure 7 shows the damping results for the full D row of whiskers.

Table 2 shows the natural resonances and the damping ratios for the D row of whiskers as calculated from the vibration experiment and the impulse experiment. Resonant frequencies agreed within $2 \%$, and damping ratios agreed within $30 \%$. Note that these are relatively large values for $\zeta$, meaning that the whiskers are very damped compared with many familiar resonant objects; typical values of $\zeta$ for musical instruments are on the order of $2-5 \times 10^{-5}$. This strong damping has significant implications for resonant oscillations during natural behavior. Most significantly, it suggests that whiskers are unlikely to bounce off of an object after contact (see case 3 below).

In all of our experiments, both on isolated whiskers and in the awake animal, the frequencies obtained are actually damped natural frequency. The damped natural frequency is related to the undamped natural frequency by the equation:

$$
f_{\text {damped }}=f_{\text {undamped }}\left(1-\zeta^{2}\right)^{1 / 2}
$$

where $\zeta$ is the damping ratio. Our whiskers typically had values of $\zeta$ between 0.11 and 0.17 (Table 2), so this will nominally lower the actual undamped natural frequencies $(<2 \%)$.

\section{Resonances during active behavior}

In the impulse experiments described previously, the base of the whisker was held rigidly fixed, and a pair of fine forceps was used to deflect the whisker. In the awake animal, however, the whiskers are actively moved back and forth near the base, and deflections occur when the whiskers make contact with objects in the environment. We wanted to determine whether resonances could be produced by interactions with objects during natural whisking behavior, and if so, how the resonant characteristics compared with those observed in the isolated whiskers. We considered five cases in which resonances might play a role in the interaction of the whiskers with the environment.

Case 1: the whisker bumps into an object and drives past it

We looked for resonant oscillations as the whiskers were actively deflected past a vertical bar, as described in Materials and Methods. A total of 19 trials were analyzed. Figure 8 shows four examples of typical whisker movement patterns that occurred as the rat drove its whiskers past the bar. In these behavioral sequences, some whiskers (those that had already passed the bar) moved freely in air, whereas other whiskers directly contacted and then deflected past the bar. This allowed us to directly compare whisker movements with and without object contact. In 10 of 19 trials, the deflected whisker exhibited one-half to one cycle of resonance before resuming the normal whisking pattern. In four cases, no clear resonance distinct from the normal whisking pattern was observable, despite clear deflection past the bar. In three trials, the whisker underwent more than two cycles of oscillation before the resonance damped out. Two trials gave ambiguous results and were not analyzed further.

Figure $8, A$ and $B$, shows two examples of the most commonly observed whisker movement pattern after deflection, in which the whisker exhibited one-half to one cycle of oscillation before resuming the normal whisking motion. The top and bottom traces of Figure $8 \mathrm{~A}$ show the angular position of two whiskers that did not make contact with the bar at any point in time during the behavioral sequence. These two whiskers show a fundamental whisking frequency of $\sim 9 \mathrm{~Hz}$. The middle trace of Figure $8 \mathrm{~A}$ shows the angular position of a third whisker ( $\sim 29 \mathrm{~mm}$ long) that did make contact with the vertical bar. The whisker remained stuck behind the bar from 0 to $17 \mathrm{msec}$, at which time it suddenly deflected past the bar (vertical arrow). It reached peak protraction at $\sim 25 \mathrm{msec}$, rebounded backwards until $32 \mathrm{msec}$, and appears to be reincorporated into the normal whisking pattern near 
A
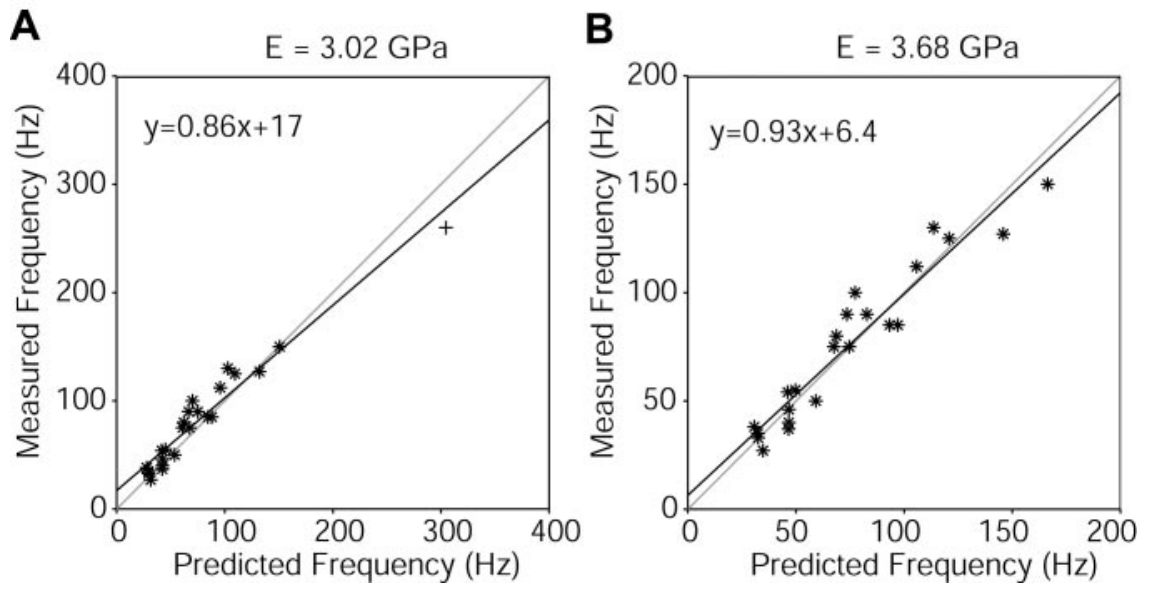

Figure 5. Experimental versus theoretical values for first-mode resonant frequencies. $A$, The best fit between predicted and measured values for the first-mode resonant frequency for all of the whiskers was found for a value of Young's modulus equal to $3.02 \mathrm{GPa}$. The line $y=x$ is shown in gray for comparison with the curve fit. An outlier at high frequency is indicated $(+) . B_{i}$ Omitting the highest frequency from the plot in $A$ yields an even better fit between predicted and measured values. In this case, the best fit was found for a value of $E=3.68 \mathrm{GPa}$. The line $y=x$ is again shown in gray for comparison with the curve fit.

\section{A}

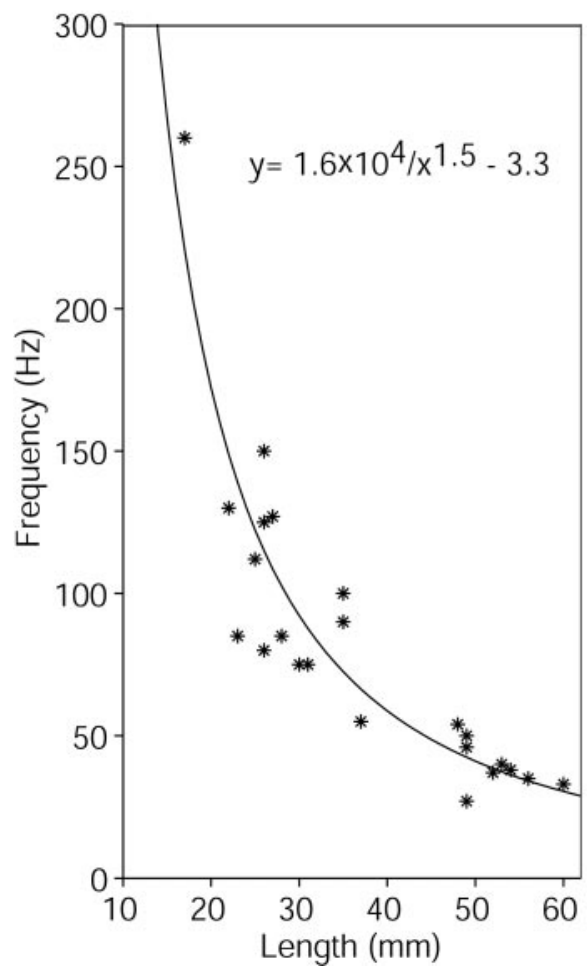

B
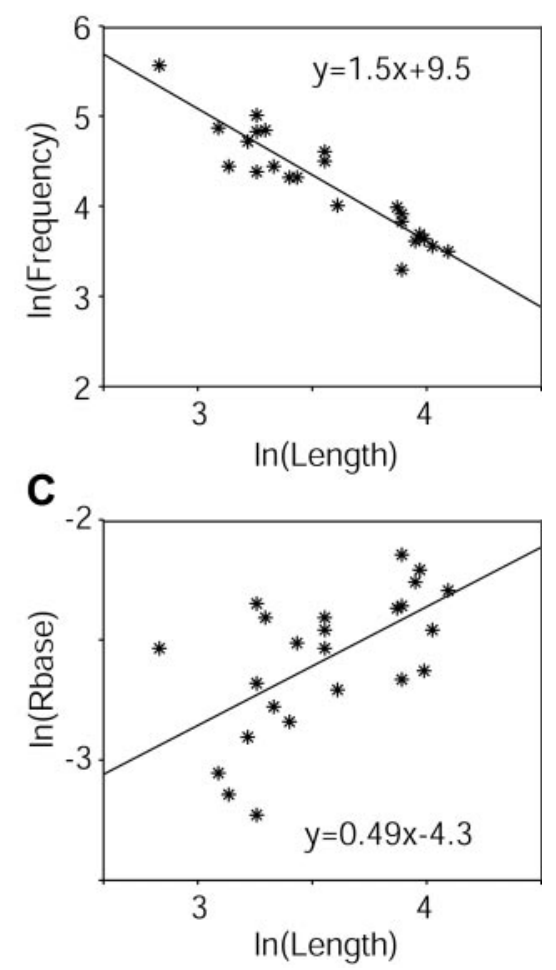

Figure 6. Resonant-frequency dependence on whisker length and base diameter. $A$, As predicted by Equation 4, the resonance frequencies are well fitted by a function that decreases inversely with the whisker length ( $L$ ). $B, A \log -\log$ plot of frequency versus length reveals a relationship of $L^{-1.5}$. C, The radius of the whisker base scales as the square root of whisker length, adding an extra 0.5 to the expected exponent of $L^{-2}$ in Equation 4.

40 msec. The half-cycle of oscillation between 25 and $32 \mathrm{msec}$ (duration, $7 \pm 1 \mathrm{msec}$; horizontal arrow) was easy to identify as a first-mode resonance in the video, and represents a resonant frequency for this whisker between 62 and $83 \mathrm{~Hz}$. To compare this experimentally measured value with theory, we used a base radius (averaged over 10 whiskers of similar length) of $0.072 \pm 0.019$ $\mathrm{mm}$, a tip radius of $0.0029 \pm 0.001 \mathrm{~mm}$, an average density of 1.14 $\mathrm{mg} / \mathrm{mm}^{3}$, and the value for Young's modulus obtained from Fig- ure $5 B(3.68 \mathrm{GPa})$. Inserting these values into Equation 4 predicts a resonantfrequency value for this whisker between 68 and $131 \mathrm{~Hz}$, centered at $107 \mathrm{~Hz}$. Some of the discrepancy between predicted and measured values is almost certainly attributable to differences in boundary conditions at the base of the whisker (see Discussion).

Figure $8 B$, middle trace, shows a second example of a whisker deflecting past the bar. In this case, the whisker was $\sim 37$ $\mathrm{mm}$ long and passed by the bar after 21 msec (vertical arrow). This whisker showed one-half cycle of oscillation between 34 and $43 \mathrm{msec}$ (duration, $9 \pm 1$ msec; horizontal arrow), representing a resonant frequency between 50 and $62 \mathrm{~Hz}$. Again using the average values for base and tip radius calculated over 10 whiskers of comparable length, theory predicts a resonant frequency for this whisker between 51 and $89 \mathrm{~Hz}$, centered at $62 \mathrm{~Hz}$. The top and bottom traces in this figure show the smooth whisking trajectory achieved by whiskers that did not hit the bar.

Figure $8 C$ illustrates the variability present in the resonant responses during natural behavior. In this example, two whiskers deflected past by the bar in quick succession (14 and $16 \mathrm{msec}$, vertical arrows). Trace 1 shows the angular position of a whisker (28 $\mathrm{mm}$ long) that underwent up to three cycles of oscillation before the resonant response damped out (lowest three horizontal arrows). Importantly, the cycles appeared to have different periods, with frequencies ranging between 41 and $55 \mathrm{~Hz}$. This suggests that the rat may be changing boundary conditions at the base of the whisker during whisker resonance. Note also that these measured values are much lower than the predicted resonant frequency, between 73 and $140 \mathrm{~Hz}$, for a whisker of this length. This large discrepancy is likely attributable to underestimation of the whisker length from foreshortening in the image, because this particular whisker appeared to be oriented at some angle extending below the image plane. In contrast to trace 1 , trace 2 shows the angular position of a whisker ( $40 \mathrm{~mm}$ long) that did not resonate in a manner distinct from smooth whisker retraction. The resonant oscillation, if it occurs, appears to be dominated by the retraction phase of the normal whisking cycle (topmost horizontal arrow). The top two traces (traces 3 and 4) of Figure $8 \mathrm{C}$ again illustrate the smooth whisking trajectory achieved by whiskers that did not hit the bar.

Finally, Figure $8 D$ shows an example of one of the most caudal whiskers (identifiable as belonging to the Greek row) deflecting past the bar near $10 \mathrm{msec}$ (vertical arrow). This whisker was over $40 \mathrm{~mm}$ in length, and shows between one-half and one full cycle 
D1
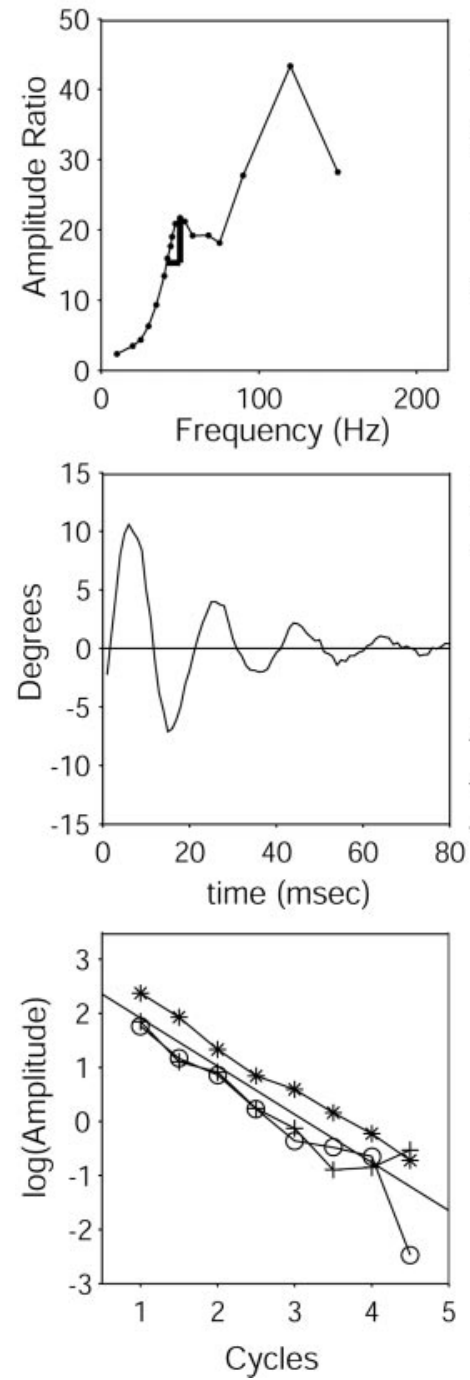

D2
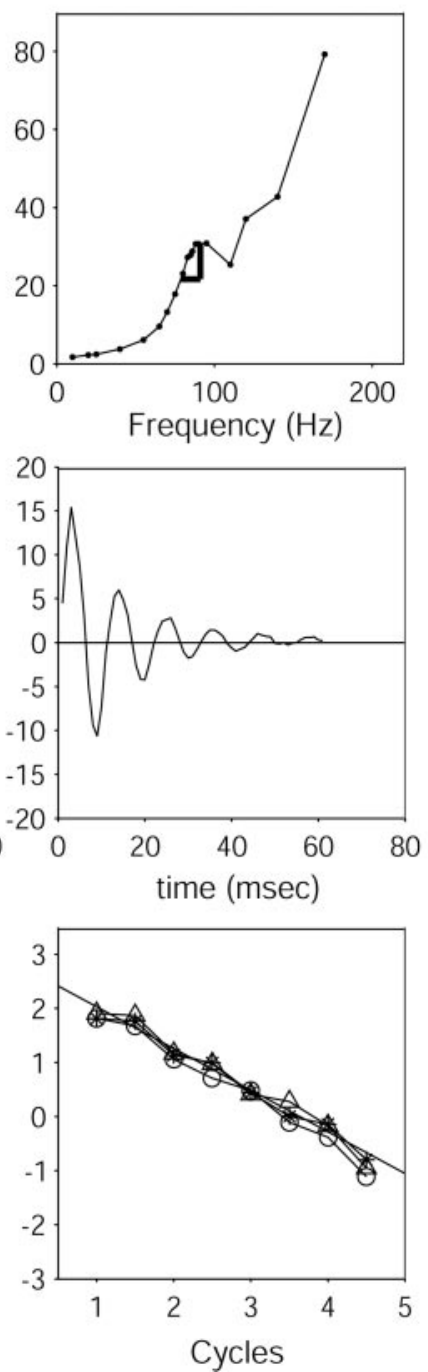

D3
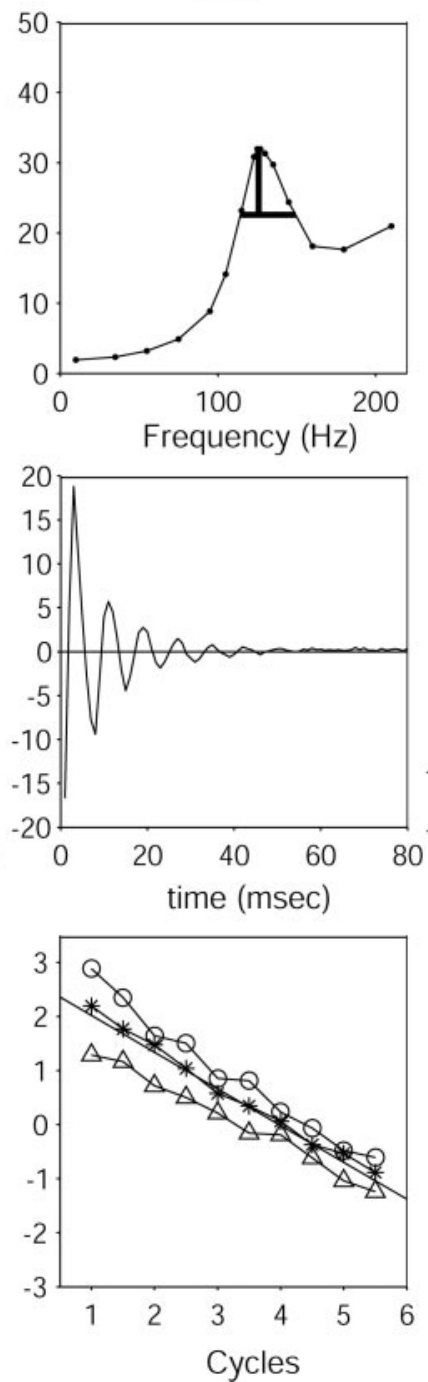

D4
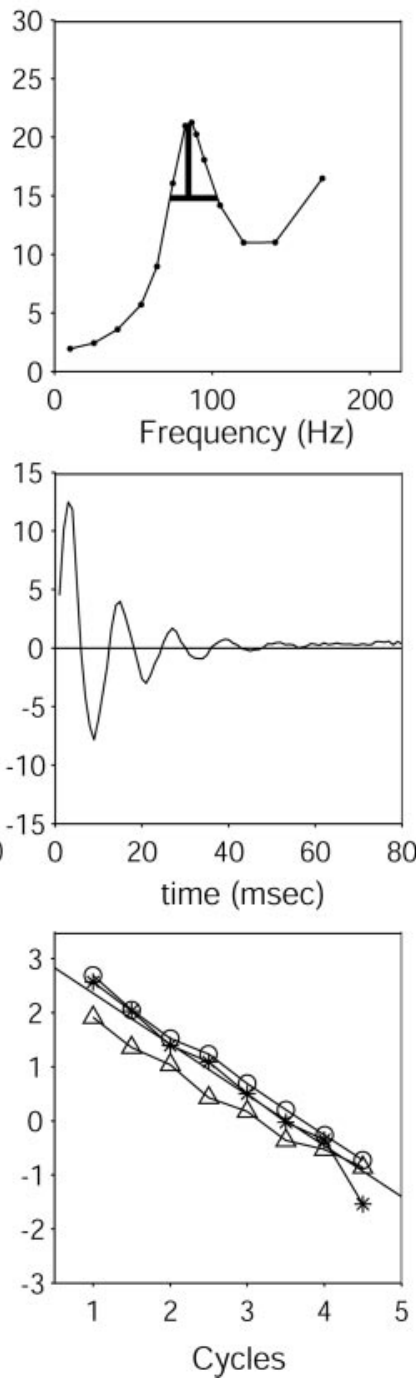

Figure 7. Damping characteristics for the D row of whiskers. Each column represents results for a different whisker (D1, D2, D3, and D4). The top row presents the resonance curve for each whisker. Damping characteristics were calculated from the width of the resonance peaks, as indicated by the gray lines. The middle row presents the results of one impulse trial for each whisker. Note that all of the whiskers are strongly damped, undergoing at most five full cycles of oscillation. The bottom row presents three log decrement curves (obtained from three impulse trials) for each whisker. Each of the three trials is indicated by a different symbol (triangles, circles, asterisks). In each graph, the $y$-axis is the log of the amplitude of the peaks and troughs of the oscillation (shown in the second row), and the $x$-axis is cycle number. The slope of the lines on these graphs is the log decrement $\Delta$.

Table 2. Measured first-mode resonant frequencies $\left(f_{1}\right)$, damping ratios $(z)$, and quality factors $(Q)$ for the whiskers of the $D$ row

\begin{tabular}{llcccccc}
\hline Whisker & Length $(\mathrm{mm})$ & $f_{1}$ vib. & $f_{1}$ imp. & $\zeta$ vib. & $\zeta$ imp. & Q vib. & Q imp. \\
\hline D1 & 49 & 50 & 50.5 & 0.17 & 0.14 & 5.9 & 7.1 \\
D2 & 35 & 91 & 89 & 0.13 & 0.12 & 7.7 \\
D3 & 27 & 126 & 128 & 0.14 & 0.11 & 7.1 \\
D4 & 28 & 85 & 85 & 0.17 & 0.15 & 5.9 & 9.0 \\
\hline
\end{tabular}

First-mode resonant frequencies $\left(f_{1}\right)$ are given in Hertz. Vib. indicates that the data were obtained from the vibration experiment. Imp. indicates that the data were obtained from the impulse experiment. $Q$ values were calculated as $Q=1 / 2 z$.

of resonance, at frequencies between 25 and $33 \mathrm{~Hz}$ (horizontal arrows). Again, the two half-cycles appear to have different periods. The top two traces show the movements of more rostral whiskers freely moving in air. Note that the caudal and rostral whiskers are moving in opposite directions over the entire duration of the whisk (100 msec).

Case 2: free whisking in air

Figure 8 shows examples of the movements of whiskers that both did and did not hit the vertical bar. As an additional control, we also tracked whisker movements during free whisking in air with no objects present, over $\sim 0.5 \mathrm{sec}$. By tracking over a longer time period, we hoped to examine the relative variation between whisks, compared with the single whisk cycles and associated resonances shown in Figure 8. This controlled for the possibility of seeing high-frequency resonances at sharp stops at the extremes of each whisking cycle. Each of the four traces in Figure 9 shows the angular position of one of the whiskers in Figure $2 B$ over time. The smooth cycles achieved by all four whiskers over 
A
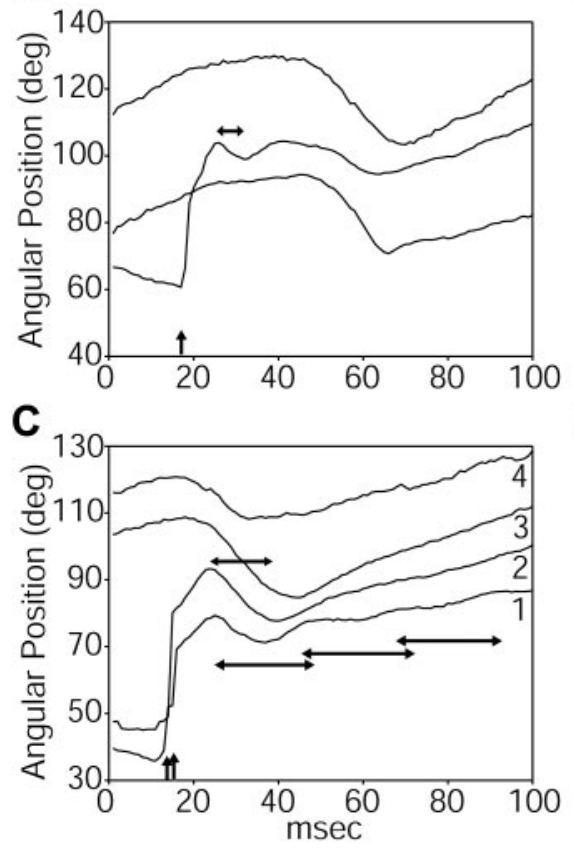

B

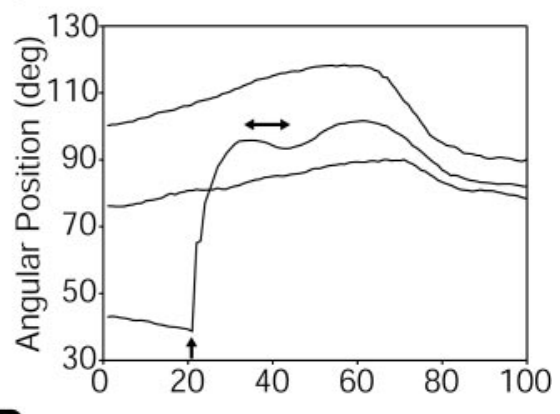

D

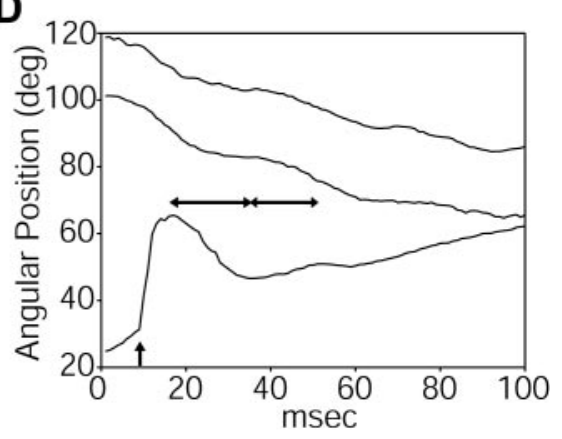

Figure 8. Resonant oscillations of the whiskers in response to deflection past a vertical bar. $A, B$, Examples of two whiskers of different lengths ( $29 \mathrm{~mm}$ in $A ; 37 \mathrm{~mm}$ in $B$ ) deflecting past the bar. The top and bottom traces in both figures show the angular position of two whiskers that did not make contact with the bar. The middle trace in each figure shows the angular position of a whisker that was stuck behind the bar and then deflected past it. Deflection times are indicated by vertical arrows. The horizontal arrows indicate the approximate half-cycle of the resonance. In $A$, the lowest trace has been offset by $-5^{\circ}$, and in $B$, the top and bottom traces have been offset by $+10^{\circ}$ and $-10^{\circ}$, respectively, for visual clarity. $C$, Whisker deflection did not always result in clear resonances. Traces 1 and 2 show the angular position of two whiskers that deflected past the bar at 14 and 16 msec (vertical arrows). In trace 2, any potential resonance exhibited immediately after deflection (top horizontal arrow) is synchronized with the fundamental whisking periodicity (compare with traces 3 and 4). In trace 1, the whisker undergoes up to three complete resonant oscillations (bottom three horizontal arrows) before damping out. Traces 3 and 4 show the angular position of two whiskers that did not make contact with the bar. Traces 3 and 1 have been offset by $+5^{\circ}$ and $-5^{\circ}$, respectively, for visual clarity. $D$, In this example, one of the most caudal whiskers deflects past the bar, and one-half to one cycle of resonance is shown. Two half-cycles of resonance are indicated by the horizontal arrows, but it is difficult to determine whether the second half-cycle constitutes resonance, active protraction, or a combination of both. Note that the caudal and rostral whiskers move in opposite directions over the duration of the whisk.

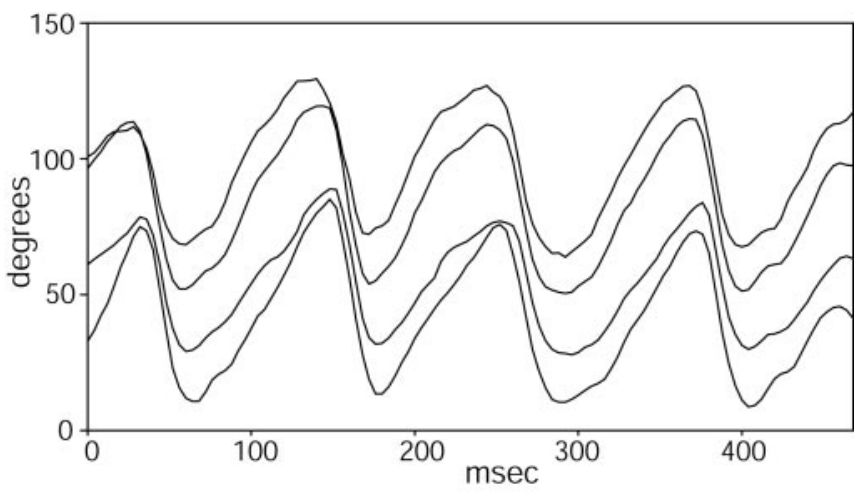

Figure 9. Whisker motion during free whisking. No resonant oscillations were observed during free whisking in air. Each trace shows the angular position of a single whisker over time. The top and bottom trace have been offset by $+10^{\circ}$ and $-10^{\circ}$, respectively, for visual clarity.

the entire duration of whisking look very similar to those shown in Figure 8 and exhibit no discernable resonant oscillations.

Thus, as expected, when the whiskers are being driven in a (relatively) steady state, at a frequency well below resonance, no resonant oscillations are present. It has also been shown that the whisking cycle has a significant dorsoventral component, so that the whisking movement actually forms a loop (Bermejo et al., 2002). It is possible that this loop helps the rat avoid the resonances that would otherwise be associated with a hard (high acceleration) stop at the end points of the whisking cycle.

Case 3: the whisker bumps into an object, and the whisker either bounces off or remains in contact with the object

In none of the 19 trials did we observe the whisker to vibrate or bounce upon its initial contact with the metal bar. Instead, after its initial contact with the bar, each whisker appeared to remain in constant direct contact with the bar as the rat drove its whiskers even further forward. As the rat protracted its whiskers more, whiskers that were stuck behind the bar deformed more and slid along the bar, until they finally deflected past the bar at their tip (Fig. 2A). Although we think it unlikely, it is conceivable that, under some behavioral conditions (e.g., very high whisking velocity), the whiskers could bounce off of an object. In this case, different modes will be excited, depending on where along its length the whisker strikes the object. Specifically, striking at different places along its length will preferentially excite modes that have amplitude maxima at the striking points.

We suggest that the high damping of the whiskers, combined with any tendency by the rat to continue to protract its whiskers after initial object contact, will in general ensure that the whiskers will stick to, instead of bounce off of, objects under exploration. Assuming that this condition approximates a fixed-fixed or fixedpinned boundary condition (see Discussion), the whisker may still resonate, but its effective length will be changed, thus altering the resonant frequency. The rat could potentially use these resonances to help determine how far away an object is. Although we never observed any resonances upon initial contact with the bar, the experiments presented here were not designed to look closely for this possibility.

\section{Case 4: texture discrimination}

The current study confirms that resonances exist in the awake, behaving animal and may enable the rat to perform fine texture discriminations as hypothesized by Neimark (2001) (for additional discussion, see Neimark et al., 2003).

\section{Case 5: whiskers held erect during head movements}

During several behaviors, including drinking and object exploration, the whiskers are often held stationary and erect while the head moves. In our behavioral videos, the tips of the whiskers were often observed to oscillate under these conditions, but these frequencies were not quantified. It is thus possible that sharp head movements may superimpose a resonance on top of the smooth whisking cycle. 


\section{Discussion}

\section{Resonances and trigeminal processing}

The main finding of this paper is that whiskers exhibit natural resonances and strong damping, both in vibration tests on isolated whiskers and in the awake, behaving animal. In the awake animal, the whiskers clearly resonate after being driven past an object (Fig. 8). We suggest below that this may be particularly important for the detection of object boundaries: high-frequency vibrational information will arise from whiskers that have deflected past an edge. The observed resonant frequencies, ranging between 27 and $260 \mathrm{~Hz}$ depending on whisker length, were well above normal whisking frequencies. These resonant properties should facilitate the transduction of high-frequency mechanical stimuli to the sensors in the whisker follicles during natural exploratory behaviors. Peripheral neurons (e.g., those in the trigeminal ganglion) might follow this high-frequency information in a one-to-one manner up to frequencies as high as $1 \mathrm{kHz}$. More central neurons are unlikely to follow one-to-one up to such high frequencies, because they will be limited by longer refractory periods. However, at higher frequencies, central neurons may track the changes in amplitude of the resonances; in other words, they may follow the envelope of the resonant signal. These two mechanisms may drive the types of high-frequency responses found in previous electrophysiological experiments (Shipley, 1974; Simons, 1978; Castro-Alamancos, 2002; Kleinfeld et al., 2002).

\section{Boundary conditions for isolated whiskers and for the behaving animal}

In the experiments on isolated whiskers, each whisker was held fixed at the base and otherwise allowed to vibrate freely, replicating the type of seismic excitation that would occur during natural whisker movement. This represents a fixed-free boundary condition and compares reasonably well with the conditions that occur as the rat moves its whiskers freely in air and makes initial contact with an object.

That the isolated whiskers were held rigidly at the base has two implications for our results. First, it means that measured values for resonant frequencies presented in this paper represent upper bounds on these frequencies, for fixed-free boundary conditions. Second, the rigid base means that damping effects were minimized, and our values of $\zeta$ thus represent lower bounds. Any decrease in how rigidly the whiskers are held at the base will decrease the natural frequencies and is likely to increase damping.

The whiskers are almost certainly held much less stiffly in the behaving animal because of compliance in the facial tissue and musculature at the whisker base. This difference in boundary conditions is likely to account for the differences observed between the isolated-whisker and awake-animal experiments: lower resonant frequencies and higher damping were observed in the awake animal than in the isolated whiskers (Figs. 7 and 8). In addition, the rat may control the compliance at the base of the whisker to actively modulate boundary conditions, thus changing both damping and resonant frequencies (see below).

Modeling the whisker base with a torsional-spring boundary condition, instead of a fixed boundary condition, might be an even better approximation to boundary whisking in air and initial object contact because of the compliance of the follicle and associated musculature. In the torsional-spring case, the boundary conditions are a mix between fixed-free and pinned-free, and to find the natural resonances, we can, to first order, interpolate between those two conditions (Gorman, 1975). In the interpolated model, the predicted fundamental frequencies would decrease by $\sim 30 \%$ (Gorman, 1975) to match our data. It may be possible to infer properties of the follicle base by using an equivalent torsional-spring constant.

Under some behavioral conditions involving object contact, such as the whisker bumping into an object and sticking against it (case $3 \mathrm{~b}$ ), the boundary conditions might be better modeled with a fixed-pinned or fixed-fixed beam. Under fixed-pinned or fixed-fixed conditions, the resonant frequencies will be higher than in the fixed-free condition, by factors of 4.39 and 6.36 , respectively. The point of contact also shortens the effective length of the whisker, further increasing the resonant frequencies. In contrast, under fixed-free boundary conditions (cases 1 , 2, 3a, and 5 in Results), the point at which the whisker contacts an object has no effect on the resonant frequencies (Morse, 1936), only on the relative amplitudes of the different spatial modes. Point of contact is also likely to be important during texture exploration (case 4) (Neimark et al., 2003).

\section{Implications for natural exploratory behavior}

\section{Experimental limitations and general observations}

In this study, we were concerned only with tracking the angular position of the whisker and examining the smoothness of the resulting trajectory for resonant frequencies. We were not concerned with tracking a particular point on the whisker, and we did not examine the dorsal-ventral component of whisker movement or resonance (Bermejo et al., 2002). Ideally, whisker movements would have been monitored in three dimensions, but we were concerned that placing any markers on the vibrissae would disrupt the natural frequencies. Instead, we were careful to examine resonances under natural conditions, with all of the whiskers intact and unaltered in any way.

In our initial analysis of the behavioral video data we observed a feature of whisking behavior that is yet undescribed in the freely behaving animal: whiskers on the same side of the face can move in opposite directions over the duration of an entire whisk. As shown in Figure 9D, the caudal whiskers can protract while the more rostral whiskers are retracting, over a time course of 100 msec. We also observed that, in general, whisking tended to be more synchronous (i.e., whiskers on the same side of the face tended to move in unison) when no obstacle was present (data not shown).

These results are consistent with, but differ slightly from, those found for rats performing a texture discrimination task (Carvell and Simons, 1990), and for rats in a head-fixed conditioned-whisking preparation (Sachdev et al., 2002). In the texture discrimination study, whiskers on the same side of the face were observed to move in opposite directions only $3 \%$ of the time, over a total of $\sim 60$ whisks (Carvell and Simons, 1990). The conditioned-whisking study reported that whiskers could move in opposite directions for durations ranging from 12 to $24 \mathrm{msec}$, most often when whiskers changed direction rapidly (Sachdev et al., 2002). Both studies reported that some whiskers could remain stationary, in continuous contact with an object, while other whiskers moved freely for several cycles (Carvell and Simons, 1990; Sachdev et al., 2002). In the present study, we found that the rostral and caudal whiskers could move in opposite directions for an entire whisk cycle (100 msec), even when only transient contact with an object occurred. The degree of independence of individual whisker movements in the freely behaving animal clearly warrants additional study.

\section{Active modulation of whisker resonances}

One of the most intriguing suggestions of our data is the possibility that the rat can actively modulate the resonant frequencies 
and damping. If the rat is able to actively change the boundary conditions at the base of the whisker, then the resonant frequencies and damping will change dramatically. In the awake animal, we observed shifts in the natural resonances over one or more cycles and also large variability in the time course of damping, dependent on the individual whisker and the phase of the whisking cycle. Both of these observations suggest that the rat may be actively changing biomechanical parameters that alter the resonances of whiskers. For example, the rat may be able to alter the resonances and time course of damping by changing muscle tension or blood flow to the follicle (Scott, 1955a,b).

Modulations of resonance are illustrated in trace 1 of Figure $8 C$ and the lowest trace of $D$, which show that the frequencies can vary over the course of one or more cycles of resonance. In addition, the time course of damping appeared to depend not only on the individual whisker but also on the phase of the whisking cycle. For example, Figure $8, A, B$, and $D$, shows that, if deflection past the bar occurs relatively early in the protraction cycle, at least one-half cycle of resonance will be present. In contrast, traces 1 and 2 of Figure $8 C$ illustrate that, if the deflection occurs later in the protraction cycle, the rat can-but does not always-incorporate the resonance into the normal retraction phase of the whisking cycle. These results suggest that the rat may at times be able to actively suppress or enhance resonant frequencies by changing the time of retraction onset.

\section{References}

Ahissar E, Sosnik R, Haidarliu S (2000) Transformation from temporal to rate coding in a somatosensory thalamocortical pathway. Nature 406:302-306.

Berg RW, Kleinfeld D (2003) Rhythmic whisking by rat: retraction as well as protraction of the vibrissae is under active muscular control. J Neurophysiol 89:104-117.

Bermejo R, Vyas A, Zeigler HP (2002) Topography of whisking. I. Monitoring of whisking movements in two dimensions. Somatosens Mot Res 19:341-346.

Bonser RHC (2000) The Young's modulus of ostrich claw keratin. J Mater Sci Lett 19:1039-1040.

Bonser RHC, Purslow PP (1995) The Young's modulus of feather keratin. J Exp Biol 198:1029-1033.

Brecht M, Preilowski B, Merzenich MM (1997) Functional architecture of the mystacial vibrissae. Behav Brain Res 84:81-97.

Carvell GE, Simons DJ (1990) Biometric analyses of vibrissal tactile discrimination in the rat. J Neurosci 10:2638-2648.

Carvell GE, Simons DJ (1995) Task- and subject-related differences in sensorimotor behavior during active touch. Somatosens Mot Res 12:1-9.

Castro-Alamancos M (2002) Different temporal processing of sensory inputs in the rat thalamus during quiescent and information processing states in vivo. J Physiol (Lond) 539:567-578.
Conway HD, Becker ECH, Dubil JF (1964) Vibration frequencies of tapered bars and circular plates. J Appl Mech 31:329-331.

Georgian J (1965) Vibration frequencies of tapered bars and circular plates. J Appl Mech 32:234-235.

Gibson JM, Welker WI (1983a) Quantitative studies of stimulus coding in first-order vibrissa afferents of rats. 1. Receptive field properties and threshold distributions. Somatosens Res 1:51-67.

Gibson JM, Welker WI (1983b) Quantitative studies of stimulus coding in first-order vibrissa afferents of rats. 2. Adaptation and coding of stimulus parameters. Somatosens Res 1:95-117.

Gorman D (1975) Free vibration analysis of beams and shafts. New York: Wiley.

Gottschaldt K, Vahle-Hinz C (1981) Merkel cell receptors: structure and transducer function. Science 214:183-186.

Hartings JA, Simons DJ (1998) Thalamic relay of afferent responses to 1- to 12-Hz whisker stimulation in the rat. J Neurophysiol 80:1016-1019.

Hartmann MJ (2001) Active sensing capabilities of the rat whisker system. Auton Robots 11:249-254.

Hartmann MJ, Bower JM (1998) Oscillatory activity in the cerebellar hemispheres of unrestrained rats. J Neurophysiol 80:1598-1604.

Kleinfeld D, Sachdev R, Merchant L, Jarvis M, Ebner F (2002) Adaptive filtering of vibrissa input in motor cortex of rat. Neuron 34:1021-1034.

Morse PM (1936) Vibration and sound. New York: McGraw-Hill.

Neimark MA (2001) The mechanics of whisking: the first stage in the transduction of surface textures into neural signals. Undergraduate Senior thesis, Princeton University.

Neimark MA, Andermann ML, Hopfield JJ, Moore CI (2003) Vibrissa resonance as a transduction mechanism for tactile encoding. J Neurosci 23:6499-6509.

O'Connor S, Berg R, Kleinfeld D (2002) Coherent electrical activity between vibrissa sensory areas of cerebellum and neocortex is enhanced during free whisking. J Neurophysiol 87:2137-2148.

Sachdev RNS, Sato T, Ebner FF (2002) Divergent movement of adjacent whiskers. J Neurophysiol 87:1440-1448.

Scott M (1955a) Blood supply of mystacial vibrissae. Nature 175:395-396.

Scott M (1955b) Blood supply of mystacial vibrissae of the rat and cat. J Anat 89:556.

Shipley MT (1974) Response characteristics of single units in the rat's trigeminal nuclei to vibrissa displacements. J Neurophysiol 37:73-90.

Simons DJ (1978) Response properties of vibrissa units in rat S1 somatosensory neocortex. J Neurophysiol 41:798-820.

Simons DJ (1985) Temporal and spatial integration in rat S1 vibrissa cortex. J Neurophysiol 54:615-635.

Sosnik R, Haidarliu S, Ahissar E (2001) Temporal frequency of whisker movement. I. Representations in brain stem and thalamus. J Neurophysiol 86:339-353.

Tongue BH (2002) Principles of vibration. New York: Oxford UP.

Vincent SB (1913) The function of the vibrissae in the behaviour of the white rat. Behav Monogr 1:1-85.

Welker WI (1964) Analysis of sniffing of the albino rat. Behaviour 22:223-244.

Zucker E, Welker WI (1969) Coding of somatic sensory input by vibrissae neurons in the rat's trigeminal ganglion. Brain Res 12:138-156. 